\title{
Diffusion Measures Indicate Fight Exposure-Related Damage to Cerebral White Matter in Boxers and Mixed Martial Arts Fighters
}

\author{
W. Shin, S.Y. Mahmoud, K. Sakaie, S.J. Banks, M.J. Lowe, M. Phillips, M.T. Modic, and C. Bernick
}

\begin{abstract}
BACKGROUND AND PURPOSE: Traumatic brain injury is common in fighting athletes such as boxers, given the frequency of blows to the head. Because DTI is sensitive to microstructural changes in white matter, this technique is often used to investigate white matter integrity in patients with traumatic brain injury. We hypothesized that previous fight exposure would predict DTI abnormalities in fighting athletes after controlling for individual variation.
\end{abstract}

MATERIALS AND METHODS: A total of 74 boxers and 81 mixed martial arts fighters were included in the analysis and scanned by use of DTI. Individual information and data on fight exposures, including number of fights and knockouts, were collected. A multiple hierarchical linear regression model was used in region-of-interest analysis to test the hypothesis that fight-related exposure could predict DTI values separately in boxers and mixed martial arts fighters. Age, weight, and years of education were controlled to ensure that these factors would not account for the hypothesized effects.

RESULTS: We found that the number of knockouts among boxers predicted increased longitudinal diffusivity and transversal diffusivity in white matter and subcortical gray matter regions, including corpus callosum, isthmus cingulate, pericalcarine, precuneus, and amygdala, leading to increased mean diffusivity and decreased fractional anisotropy in the corresponding regions. The mixed martial arts fighters had increased transversal diffusivity in the posterior cingulate. The number of fights did not predict any DTI measures in either group.

CONCLUSIONS: These findings suggest that the history of fight exposure in a fighter population can be used to predict microstructural brain damage.

ABBREVIATIONS: TBI = traumatic brain injury; FA = fractional anisotropy; $L D=$ longitudinal diffusivity; TD = transversal diffusivity; MD = mean diffusivity

$\mathbf{T}$ raumatic brain injury (TBI) has been reported in athletes involved in combat sports who are frequently exposed to repetitive blows to the head, such as boxers. ${ }^{1-8}$ This cumulative head trauma is thought to cause chronic traumatic encephalopathy as a result of chronic axonal injury. ${ }^{9}$ The clinical syndrome related to chronic traumatic encephalopathy in boxing is characterized by impulsive behavior, cognitive dysfunction, and in some cases, violence or suicide. $^{10}$

Conventional MR imaging, such as T1-weighted and T2weighted anatomic scans, cannot assess mild white matter disruption. Because DTI is sensitive to microstructural changes in white matter, this technique is often used to investigate white matter

Received December 21, 2012; accepted after revision May 10, 2013.

From the Imaging Institute (W.S., S.Y.M., K.S., M.J.L., M.P.) and Neurological Institute (C.B.), Cleveland Clinic, Cleveland, Ohio; and Lou Ruvo Center for Brain Health (S.J.B., M.T.M.), Cleveland Clinic, Las Vegas, Nevada.

Please address correspondence to Wanyong Shin, PhD, Imaging Institute, 9500 Euclid Ave, Mail Code U-15, Cleveland, OH 44195; e-mail: wanyong.shin@gmail.com http://dx.doi.org/10.3174/ajnr.A3676 integrity in patients with TBI. ${ }^{11-17}$ Previous studies have found that fractional anisotropy (FA) values in the corpus callosum, ${ }^{14,16,17}$ internal capsule, ${ }^{11,14,16,17}$ cingulum, ${ }^{14}$ and centrum semiovale $^{11,14,16,17}$ are decreased in patients with TBI versus healthy control subjects. A longitudinal TBI study found that FA was decreased in the white matter of study participants because of decreased longitudinal diffusivity (LD) and increased transversal diffusivity (TD). ${ }^{13}$ Another study demonstrated that the peaks of whole-brain ADC histograms were significantly correlated with scores on the Glasgow Coma Scale in patients with TBI, indicating that a change in DTI values can predict functional deficit. ${ }^{18}$

A limited number of studies have investigated diffusion changes in the white matter of fighting athletes. ${ }^{7,8,19}$ These studies found that among fighting athletes, whole-brain diffusion is increased, ${ }^{8}$ FA is reduced in the genu and splenium of the corpus callosum and the posterior internal capsule, ${ }^{7}$ and FA is reduced and ADC increased in the lower brain, the splenium of the corpus callosum, and the lateral and dorsolateral cortical regions. ${ }^{19} \mathrm{Al}-$ though these studies used age- and sex-matched control groups, 
Table 1: Pearson correlation between predictors

\begin{tabular}{|c|c|c|c|c|c|c|}
\hline & Age & Years of Education & Weight & Number of Knockouts & Number of Fights & Years of Fighting \\
\hline Age & & $0.288^{b}$ & $0.268^{\mathrm{a}}$ & $0.321^{b}$ & 0.092 & $0.511^{\mathrm{b}}$ \\
\hline Years of education & & & $0.283^{\mathrm{b}}$ & -0.009 & 0.013 & 0.055 \\
\hline Weight & & & & 0.061 & 0.025 & 0.010 \\
\hline Number of knockouts & & & & & 0.057 & $0.250^{\mathrm{a}}$ \\
\hline $\begin{array}{l}\text { Number of fights } \\
\text { Years of fighting }\end{array}$ & & & & & & $0.520^{b}$ \\
\hline
\end{tabular}

${ }^{a}$ Correlation is significant at the .01 level (2-tailed).

${ }^{b}$ Correlation is significant at the .001 level (2-tailed).

variations among fighters in number of fights and number of knockouts have not been fully investigated. Zhang et $\mathrm{al}^{8}$ found that the number of hospitalizations for boxing injuries was positively correlated with the peak of the Gaussian-fitted brain tissue compartment in a whole-brain diffusion histogram in 24 professional boxers. However, this finding did not offer information regarding which regions were affected in hospitalized boxers.

In the present study, we sought to assess previous fight history and regional MR diffusion parameters to evaluate the relationship between microstructural brain damage and fight-related exposure. We hypothesized that previous fight exposure would predict DTI changes suggestive of microstructural damage in fighter populations.

\section{MATERIALS AND METHODS Fighter Population}

The Professional Fighters' Brain Health Study was approved by the local institutional review board, and all participants provided informed consent. Participants in the Professional Fighters' Brain Health Study are athletes ages 18 and older who have achieved at least a fourth-grade reading level and are licensed in Nevada to fight professionally in one of the combat sports (boxing, mixed martial arts). In the Professional Fighters' Brain Health Study protocol, participants are scanned for a baseline evaluation and then annually over 4 years. Eligible participants in the current study had no MR-visible central nervous system disease or neurologic disorder.

Data from the first 199 professional fighters who visited for the baseline evaluation were used for this study. Information on sex, age, weight, years of education, type of fighting (boxing or mixed martial arts), years of fighting, number of fights, and number of knockouts was collected for each participant. Fifteen female fighters were excluded from the study to eliminate sex effects, and a 71-year-old male fighter was also excluded because his age made him an outlier. Missing data of individual information were deleted list-wise from 183 fighters in the analysis. A total of 155 male fighters (74 boxers and 81 mixed martial arts fighters) were included in the final analysis (Table 1).

\section{MR Protocols}

MR images were performed on a 3T Verio scanner with a 32channel head coil (Siemens, Erlangen, Germany). 3D T1weighted scans $\left(\right.$ voxel size $=1 \times 1 \times 1.2 \mathrm{~mm}^{3}$; TR/TE/TI $=$ 2300/2.98/900 ms; flip angle $=9^{\circ}$; scan time $\left.=9: 14\right)$, T2-weighted scans $\left(\right.$ voxel size $=0.8 \times 0.8 \times 4 \mathrm{~mm}^{3} ; \mathrm{TR} / \mathrm{TE}=5000 / 84 \mathrm{~ms} ; 38$ sections; scan time $=0: 57$ ), and FLAIR scans (voxel size $=0.8 \times$ $0.8 \times 4 \mathrm{~mm}^{3}$; TR/TE/TI $=7000 / 81 / 2220 \mathrm{~ms} ; 38$ sections; scan time $=2: 36$ ) were performed. A single-shot EPI scan was used to acquire diffusion tensor mapping $(\mathrm{TR} / \mathrm{TE}=7000 / 91 \mathrm{~ms}$ FOV $=$ $240 \times 240 \mathrm{~mm}^{2}$; voxel size $=2.5 \times 2.5 \times 2.5 \mathrm{~mm}^{3} ; 49$ axial sections with no gap between sections; partial Fourier factor $=$ 5/8; NEX $=1 ; 71$ nonlinear diffusion-weighting gradients with $\mathrm{b}$-value $=1000$ seconds $/ \mathrm{mm}^{2}$ and eight $b=0$ volumes for averaging; scan time $=8: 24$ ).

\section{DTI Postprocessing}

Field map-based distortion correction ${ }^{20}$ was applied in the timeseries diffusion images to unwarp EPI geometric distortion, and an iterative motion and eddy current artifact correction method was used $^{21}$ before DTI parameter calculation. Subsequently, the diffusion tensor was calculated at each voxel with log-linear ordinary least squares. ${ }^{22}$ The tensors were diagonalized, yielding eigenvalues from which $\mathrm{LD}, \mathrm{TD}$, mean diffusivity (MD), and FA were calculated with the use of in-house software. ${ }^{23}$ The detailed definition of DTI parameters is described with 3 eigenvectors $\left(\lambda_{1,2,3}\right.$, and $\left.\lambda_{1} \geq \lambda_{2} \geq \lambda_{3}\right)$; $\mathrm{LD}=\lambda_{1}, \mathrm{TD}=\left(\lambda_{2}+\lambda_{3}\right) / 2, \mathrm{MD}=$ $\left(\lambda_{1}+\lambda_{2}+\lambda_{3}\right) / 3$, and FA $=\left\{\left(\left(\lambda_{1}-\lambda_{2}\right)^{2}+\left(\lambda_{2}-\lambda_{3}\right)^{2}+\right.\right.$ $\left.\left.\left(\lambda_{3}-\lambda_{1}\right)^{2}\right) /\left(\lambda_{1}^{2}+\lambda_{2}^{2}+\lambda_{3}^{2}\right) / 2\right\}^{1 / 2}$.

\section{ROI Analysis}

Seventy-three ROIs in white matter ( 3 corpus callosum areas and 35 white matter regions in each hemisphere) and 7 subcortical gray matter regions in each hemisphere were defined in T1weighted image space with the use of the FreeSurfer software package (http://surfer.nmr.mgh.harvard.edu/). The defined ROIs were aligned in DTI space by use of the Linear Image Registration Tool FLIRT (http://www.fmrib.ox.ac.uk/). ${ }^{24}$ Average DTI values were calculated in each ROI. After a visual check, the caudal middle frontal, lateral orbitofrontal, medial orbitofrontal, parsorbitalis, rostral middle frontal, superior frontal, and frontal pole regions were excluded because of imperfect coregistration between the anatomic image and the unwarped EPI image, caused by EPI geometric distortion in the frontal lobe area.

\section{Statistical Analysis}

Pearson correlation was calculated for fighter information (age, years of education, weight, number of knockouts, number of fights, and years of fighting). Years of fighting was highly correlated with age (correlation $=0.511 ; P<.001$ ) and with number of fights (correlation $=0.520 ; P<.001$ ); therefore, number of knockouts and number of fights were selected as fight-related predictors. Age, weight, years of education, number of knockouts, and number of fights were chosen as predictors to represent variance in independent measures (Table 1).

Multiple hierarchical linear regression analyses were performed to test the hypothesis that fight-related exposure (number 
of knockouts and number of fights) could predict average DTI values in different regions. Age, weight, and years of education were controlled to ensure that they would not account for the hypothesized effects. Therefore, model 1 used age, weight, and years of education as predictors for DTI value $(\mathrm{DTI}=$ constant + $\mathrm{a}_{1} \times$ age $+\mathrm{a}_{2} \times$ weight $+\mathrm{a}_{3} \times$ years of education). Model $2 \mathrm{~A}$ used age, weight, years of education, and number of knockouts as predictors $\left(\right.$ DTI $=$ constant $+b_{1} \times$ age $+b_{2} \times$ weight $+b_{3} \times$ years of education $+\mathrm{b}_{4} \times$ number of knockouts), and model $2 \mathrm{~B}$

Table 2: Demographic data

\begin{tabular}{|c|c|c|}
\hline & Boxers & Mixed Marital Arts Fighters \\
\hline Age, $y$ & $28.0 \pm 6.3$ & $28.2 \pm 4.8$ \\
\hline Years of education $^{a}$ & $12.7 \pm 3.0$ & $14.0 \pm 2.4$ \\
\hline Weight, kg & $74.5 \pm 15.8$ & $78.7 \pm 10.9$ \\
\hline Number of fights & $13.2 \pm 15.7$ & $12.1 \pm 14.1$ \\
\hline Number of knockouts & $1.1 \pm 2.0$ & $1.0 \pm 2.1$ \\
\hline
\end{tabular}

${ }^{a}$ Significantly different between groups $(P<.01)$.

Table 3: Areas in which number of knockouts predicted DTI values after controlling for individual variation

\begin{tabular}{|c|c|c|c|c|c|c|}
\hline \multirow[b]{2}{*}{ ROI } & \multicolumn{4}{|c|}{ Boxers } & \multicolumn{2}{|c|}{ Mixed Martial Arts Fighters } \\
\hline & LD & TD & MD & FA & TD & FA \\
\hline Corpus callosum posterior & & $\mathrm{a}$ & & $\mathrm{b}$ & & $\mathrm{a}$ \\
\hline Corpus callosum central & & a & a & & & \\
\hline Cuneus & & & & $L^{a}$ & & \\
\hline Inferior parietal & $\mathrm{L}^{\mathrm{a}}$ & & & & & \\
\hline Isthmus cingulate & $L^{c}, R^{b}$ & $L^{c}, R^{c}$ & $\mathrm{~L}^{\mathrm{c}}, \mathrm{R}^{\mathrm{c}}$ & & & \\
\hline Pars triangularis & $\mathrm{L}^{\mathrm{a}}$ & & & & & \\
\hline Pericalcarine & $L^{b}, R^{a}$ & $L^{c}, R^{b}$ & $\mathrm{~L}^{\mathrm{c}}, \mathrm{R}^{\mathrm{b}}$ & & & \\
\hline Posterior cingulate & & & & & $\mathrm{R}^{\mathrm{a}}$ & \\
\hline Precuneus & $\mathrm{R}^{\mathrm{a}}$ & $\mathrm{R}^{\mathrm{a}}$ & $L^{a}, R^{a}$ & & & \\
\hline Caudate & & & & $L^{a}$ & & \\
\hline Putamen & & & & $L^{a}, R^{a}$ & & \\
\hline Pallidum & $L^{a}, R^{b}$ & $L^{a}, R^{a}$ & $L^{a}, R^{b}$ & & & \\
\hline Amygdala & $\mathrm{L}^{\mathrm{a}}, \mathrm{R}^{\mathrm{c}}$ & $\mathrm{R}^{\mathrm{b}}$ & $L^{a}, R^{c}$ & & & \\
\hline
\end{tabular}

Note: $-L$ and $R$ represent the left and right hemispheres, respectively.

a $P<.05$.

$\mathrm{b} P<.01$.

${ }^{c} p<.001$.

Table 4: Coefficients for number of knockouts for the areas in which number of knockouts predicted DTI values after controlling for individual variation

\begin{tabular}{|c|c|c|c|c|c|c|}
\hline \multirow[b]{2}{*}{ ROI } & \multicolumn{4}{|c|}{ Boxers } & \multicolumn{2}{|c|}{$\begin{array}{c}\text { Mixed Martial Arts } \\
\text { Fighters }\end{array}$} \\
\hline & LD & TD & MD & FA & TD & FA \\
\hline $\begin{array}{l}\text { Corpus callosum posterior } \\
\text { Corpus callosum central } \\
\text { Cuneus } \\
\text { Inferior parietal } \\
\text { Isthmus cingulate } \\
\text { Pars triangularis } \\
\text { Pericalcarine } \\
\text { Posterior cingulate } \\
\text { Precuneus } \\
\text { Caudate } \\
\text { Putamen } \\
\text { Pallidum } \\
\text { Amygdala }\end{array}$ & $\begin{array}{l}\text { (L) } 6.56 \\
\text { (L) } 24.24 \\
\text { (R) } 16.73 \\
\text { (L) }-7.61 \\
\text { (L) } 15.80 \\
\text { (R) } 10.05 \\
\text { (R) } 8.34\end{array}$ & $\begin{array}{l}26.48 \\
20.28 \\
\text { (L) } 19.25 \\
\text { (R) } 22.61 \\
\text { (L) } 24.26 \\
\text { (R) } 15.92 \\
\text { (R) } 9.90\end{array}$ & $\begin{array}{l}18.19 \\
\text { (L) } 20.91 \\
\text { (R) } 20.65 \\
\text { (L) } 21.44 \\
\text { (R) } 13.96 \\
\text { (L) } 6.63 \\
\text { (R) } 9.38\end{array}$ & $\begin{array}{c}-18.9 \\
(L)-4.60\end{array}$ & (R) 5.49 & -7.27 \\
\hline
\end{tabular}

Note: - L and R represent the left and right hemispheres, respectively A coefficient unit is $10^{-6} \mathrm{~mm}^{2} / \mathrm{s}$ for $L D$, TD, and $M D$, and $10^{-3}$ for FA. used age, weight, years of education, and number of fights as predictors $\left(\mathrm{DTI}=\right.$ constant $+\mathrm{c}_{1} \times$ age $+\mathrm{c}_{2} \times$ weight $+\mathrm{c}_{3} \times$ years of education $+c_{4} \times$ number of fights). Regions were defined as activated (ie, a fight-related exposure predicted DTI values) when the following criteria were met: 1) the significance change from model 1 to model $2 \mathrm{~A}$ or $2 \mathrm{~B}$ was significant, and 2) the linear regression result of model $2 \mathrm{~A}$ or $2 \mathrm{~B}$ was significant.

\section{RESULTS}

We found no significant $(P>.05)$ differences between boxers and mixed martial arts fighters in age, weight, number of fights, or number of knockouts, but there was a significant difference between the groups in years of education $(P=.0045$; Table 2$)$.

The number of knockouts among boxers predicted increased $\mathrm{LD}$ in the inferior parietal, isthmus cingulate, pericalcarine, precuneus, and amygdala areas and increased TD in the middle and posterior corpus callosum, isthmus cingulate, pericalcarine, precuneus, and amygdala regions after we controlled for individual variations in age, weight, and years of education (Table $3)$. There was increased MD and decreased FA in the corresponding areas. Interestingly, in mixed martial arts fighters, the number of knockouts predicted only decreased FA in the posterior corpus callosum and increased TD in the posterior cingulate. In the posterior corpus callosum of both boxers and mixed martial arts fighters, the number of knockouts predicted decreasing FA, but the decreasing tendency or the magnitude of coefficient of the number of knockouts over FA was larger in boxers than in mixed martial arts fighters $\left(-18.90 \times 10^{-6} \mathrm{~mm}^{2} / \mathrm{s}\right.$ versus $-7.27 \times 10^{-6} \mathrm{~mm}^{2} / \mathrm{s}$; Table 4$)$.

The number of knockouts predicted decreased LD in the pars triangularis, decreased LD and TD in the pallidum, and increased FA in the caudate and putamen regions. The number of fights did not predict DTI values in boxers or mixed martial arts fighters. The average DTI values for the regions in which the number of knockouts predicted DTI values are presented in Table 5.

\section{DISCUSSION}

We found that the number of knockouts in fighting athletes, most prominently in the boxing group, predicted DTI changes after we controlled for age, weight, and education effects. This finding suggests that the number of knockouts experienced by boxers can predict microstructural damage in the brain, as represented by increased $\mathrm{LD}$ and TD. In contrast, the number of fights did not account for microstructural injury. 
Table 5: Average DTI values for the areas in which number of knockouts predicted DTI values

\begin{tabular}{|c|c|c|c|c|c|c|}
\hline \multirow[b]{2}{*}{ ROI } & \multicolumn{4}{|c|}{ Boxers } & \multicolumn{2}{|c|}{$\begin{array}{l}\text { Mixed Martial } \\
\text { Arts Fighters }\end{array}$} \\
\hline & LD & TD & MD & FA & TD & FA \\
\hline Corpus callosum posterior & 1.617 & 0.689 & 0.998 & 0.525 & 0.656 & 0.546 \\
\hline Corpus callosum central & 1.533 & 0.679 & 0.964 & 0.506 & 0.653 & 0.512 \\
\hline Cuneus & 0.966 & 0.727 & 0.807 & 0.189 & 0.712 & 0.194 \\
\hline Inferior parietal & 1.007 & 0.657 & 0.774 & 0.280 & 0.648 & 0.284 \\
\hline Isthmus cingulate & 1.292 & 0.569 & 0.810 & 0.481 & 0.540 & 0.498 \\
\hline Pars triangularis & 0.956 & 0.650 & 0.752 & 0.266 & 0.635 & 0.270 \\
\hline Pericalcarine & 1.128 & 0.787 & 0.901 & 0.238 & 0.769 & 0.250 \\
\hline Posterior cingulate & 1.209 & 0.570 & 0.783 & 0.448 & 0.543 & 0.463 \\
\hline Precuneus & 1.086 & 0.647 & 0.793 & 0.326 & 0.631 & 0.337 \\
\hline Caudate & 1.235 & 0.966 & 1.056 & 0.170 & 0.949 & 0.168 \\
\hline Putamen & 0.879 & 0.610 & 0.700 & 0.240 & 0.602 & 0.246 \\
\hline Pallidum & 0.808 & 0.515 & 0.613 & 0.323 & 0.518 & 0.315 \\
\hline Amygdala & 1.125 & 0.864 & 0.951 & 0.181 & 0.854 & 0.184 \\
\hline
\end{tabular}

Note:-A coefficient unit is $10^{-3} \mathrm{~mm}^{2} / \mathrm{s}$ for $L D, T D$, and $M D$ and $0-1$ for FA.

We found that increased TD resulted in increased MD and decreased FA in the corpus callosum among boxers. The corpus callosum is an area known to be involved in TBI. ${ }^{7,16,17,25-30} \mathrm{Sev-}$ eral studies have demonstrated increased MD and/or decreased FA in various white matter regions among patients with mild TBI versus matched control subjects. ${ }^{7,17,31}$ Inglese et al $^{17}$ found a significant reduction in FA in the corpus callosum, internal capsule, and centrum semiovale and a significant increase in MD in the corpus callosum and internal capsule in 46 patients with mild TBI. In a study of 49 professional boxers who had been exposed to repeated blows to the head, Zhang et $\mathrm{al}^{7}$ observed a significant decrease in FA in the anterior and posterior corpus callosum, as well as a significant decrease in FA and an increase in MD in the internal capsule. In a study of 24 boxers, Zhang et $\mathrm{al}^{8}$ also found that the mean diffusion constant of the brain tissue was significantly correlated with the times of hospitalization for boxing injuries $(P<.05)$ but not with age when boxing was started, total rounds, years of fighting, or number of wins and losses. Cubon et $\mathrm{al}^{31}$ reported that college athletes with mild TBI ( 5 men and 5 women) who had concussion showed significantly increased MD in the left hemisphere, spanning the sagittal striatum, retrolenticular section of the internal capsule, and the posterior thalamic radiation (by use of tract-based spatial statistics), but there was no significant change in FA. In a recent study, Zhang et $\mathrm{al}^{32}$ found no significant differences in fMRI and DTI results among patients with mild TBI versus control subjects; the authors suggested that these regional variations in FA and $\mathrm{MD}$ changes in patients with mild TBI might be the result of differing timeframes, technological issues involved in DTI, and different methodologic approaches.

Besides the corpus callosum, the areas in which increased LD was predicted by the number of knockouts among boxers were identical to the regions in which increased TD was predicted by the number of knockouts with the exception of the inferior parietal region. Our results of increased LD and TD among boxers suggests that knockouts could induce irreversible myelin damage, as has been discussed previously. ${ }^{30}$ Kraus et $\mathrm{al}^{30}$ found that patients with mild to severe TBI showed increased TD and LD in all white matter regions; patients with mild TBI also showed increased LD in the sagittal striatum and superior longitudinal fasciculus but not TD in any regions versus control subjects. This study also found that moderate to severe TBI groups showed severe impairment of neuropsychological assessment, compared with mild TBI and control groups. We have performed cognitive testing for the participants in our study, and further analyses will be conducted to assess whether our DTI findings are correlated with individual cognitive assessments.

In our study, decreased LD and/or TD in the pars triangularis and pallidum were predicted by the number of knockouts. In a previous study, Bazarian et $\mathrm{al}^{33}$ found decreased median MD and increased median FA in the posterior corpus callosum in 6 patients with mild TBI when scans were performed within 4 hours after the injury; MD was also decreased in the left anterior internal capsule in ROI analysis. Wilde et $\mathrm{al}^{34}$ found increased FA in adolescents with mild TBI who were scanned within an average of 2.7 days (1-6 days) after the injury. McAllister et $\mathrm{al}^{35}$ showed that strain rate positively predicted FA changes before and 10 days after head injury in the corpus callosum in 10 athletes. Mayer et $\mathrm{al}^{36}$ also found increased FA in patients with mild TBI during the semi-acute injury period (average of 12 days after injury) and confirmed this finding in a replicate experiment (average of 15.6 days after injury). ${ }^{37}$ This increased FA in semi-acute mild TBI may be induced by inflammation and/or cytotoxic edema and could be transient. A recent study by Wilde et $\mathrm{al}^{38}$ assessed FA values in patients with mild TBI over time during the semi-acute period (from day 1 to days 7-8 after injury). Our study did not consider time after the onset of head injury (eg, the latest knockout). However, boxers who are exposed to repeated blows to the head, should be considered separately from patients with mild TBI, who generally have a single incidence of head injury. The decreased LD and TD among boxers in our study might be related to this repeated pattern of brain tissue injury and recovery. Further studies are needed to assess this possibility.

Although boxers in our study had increased LD and TD in various white matter regions predicted by number of knockouts, mixed martial arts fighters had decreased FA in the posterior corpus callosum and increased TD in the posterior cingulate region only predicted by the number of knockouts. Furthermore, when considering the posterior corpus callosum where the number of knockouts predicted FA in both boxers and the mixed martial arts fighters, the higher magnitude of coefficient for the number of knockouts over FA in boxers versus mixed martial arts fighters was 
observed $\left(-18.90 \times 10^{-6} \mathrm{~mm}^{2} /\right.$ s versus $\left.-7.27 \times 10^{-6} \mathrm{~mm}^{2} / \mathrm{s}\right)$. This may reflect the different natures of boxing and mixed martial arts. Boxers mainly target the head of their opponent, which exposes the boxers to a higher risk of repetitive head injuries; mixed martial arts fighters use striking and grappling techniques.

DTI is known to be affected by a patient sex, ${ }^{39}$ age, ${ }^{39-42}$ intelligence level, ${ }^{43,44}$ and body weight. ${ }^{45}$ We therefore included only male fighters in our study and used multiple hierarchical linear regression analyses to control for age, years of education, and weight. Although we assumed that body weight was an individual factor for the purpose of this analysis, it is possible that weight plays a role in the severity of brain injury, as a blow from a heavy-weight fighter would be expected to cause more damage than a blow from a light-weight fighter. In future research, weight class should be considered as an individual factor. We also plan to recruit an age- and educationmatched control group for future studies of longitudinal MR changes, but we believe that our findings in the current study are not limited by the absence of a control group.

A previous study suggested that sports-related concussions are often unreported by athletes. ${ }^{46}$ For this study, we used the number of knockouts in both the amateur and professional career periods, and the number of knockouts includes not only the loss of consciousness, leading to down more than 10 counts, but also the technical knockout including referee stop, corner stop, and multiple knockdowns caused by lack of the self-defense ability. The information of the number of knockouts from professional fights was collected and verified from the fighters' official log, whereas the information from amateur fights was collected through a self-reporting questionnaire. Inaccuracy in self-reporting may therefore be a confounding variable. Additionally, there probably are differences in the threshold of trauma required to cause a knockout in individuals, a factor we plan to address in future research.

This study involved data from one time point, but we intend to follow these fighters for at least 4 years. Although we do not know which, if any, of these fighters will have development of chronic traumatic encephalopathy, the results of the current study suggest that measurable microstructural changes or diffusion metrics can be predicted by history of repeated head injuries or severity in fighters' careers after controlling for individual variations.

\section{ACKNOWLEDGMENTS}

This work was supported by the Imaging Institute, Cleveland Clinic. The authors gratefully acknowledge Megan Griffiths for editing the manuscript and Dr HangJoon Jo for the helpful discussion of the data analysis. The authors also thank MR imaging technologist Trish Lake for technical support and research assistants Triny Cooper and Isaac Santa Ana for their work.

Disclosures: Wanyong Shin-RELATED: Grant: Lincy Foundation.* Ken SakaieUNRELATED: Grants/Grants Pending: NIH, NMSS, DOD. Mark Lowe-RELATED: Grant: Lincy Foundation.* Michael Phillips—RELATED: Grant: Lincy Foundation.* Charles Bernick—RELATED: Grant: Lincy Foundation*; UNRELATED: Consultancy: Bayer; Payment for Lectures (including service on speakers bureaus): Novartis, UCB, Teva (*money paid to institution).

\section{REFERENCES}

1. Bledsoe GH, Li G, Levy F. Injury risk in professional boxing. South Med J 2005;98:994-98
2. Blonstein JL. Letter: Traumatic encephalopathy in a young boxer. Lancet 1974;2:1213

3. Guterman A, Smith RW. Neurological sequelae of boxing. Sports Med 1987;4:194-210

4. Handratta V, Hsu E, Vento J, et al. Neuroimaging findings and brain-behavioral correlates in a former boxer with chronic traumatic brain injury. Neurocase 2010;16:125-34

5. Harvey PK, Davis JN. Traumatic encephalopathy in a young boxer. Lancet 1974;2:928-29

6. Saing T, Dick MC, Nelson PT, et al. Frontal cortex neuropathology in dementia pugilistica. J Neurotrauma 2012;29:1054-70

7. Zhang L, Heier LA, Zimmerman RD, et al. Diffusion anisotropy changes in the brains of professional boxers. AJNR Am J Neuroradiol 2006;27:2000-04

8. Zhang L, Ravdin LD, Relkin N, et al. Increased diffusion in the brain of professional boxers: a preclinical sign of traumatic brain injury? AJNR Am J Neuroradiol 2003;24:52-57

9. McKee AC, Cantu RC, Nowinski CJ, et al. Chronic traumatic encephalopathy in athletes: progressive tauopathy after repetitive head injury. J Neuropathol Exp Neurol 2009;68:709-35

10. Mendez MF. The neuropsychiatric aspects of boxing. Int J Psychiatr Med 1995;25:249-62

11. Yuan W, Holland SK, Schmithorst VJ, et al. Diffusion tensor MR imaging reveals persistent white matter alteration after traumatic brain injury experienced during early childhood. AJNR Am J Neuroradiol 2007;28:1919-25

12. Wu TC, Wilde EA, Bigler ED, et al. Longitudinal changes in the corpus callosum following pediatric traumatic brain injury. Dev Neurosci 2010;32:361-73

13. Sidaros A, Engberg AW, Sidaros K, et al. Diffusion tensor imaging during recovery from severe traumatic brain injury and relation to clinical outcome: a longitudinal study. Brain 2008;131:559-72

14. Rutgers DR, Toulgoat F, Cazejust J, et al. White matter abnormalities in mild traumatic brain injury: a diffusion tensor imaging study. AJNR Am J Neuroradiol 2008;29:514-19

15. Matthews PM. Brain imaging of multiple sclerosis: the next 10 years. Neuroimaging Clin North Am 2009;19:101-12

16. Xu J, Rasmussen IA, Lagopoulos J, et al. Diffuse axonal injury in severe traumatic brain injury visualized using high-resolution diffusion tensor imaging. J Neurotrauma 2007;24:753-65

17. Inglese M, Makani S, Johnson G, et al. Diffuse axonal injury in mild traumatic brain injury: a diffusion tensor imaging study. J Neurosurg 2005;103:298-303

18. Shanmuganathan K, Gullapalli RP, Mirvis SE, et al. Whole-brain apparent diffusion coefficient in traumatic brain injury: correlation with Glasgow Coma Scale score. AJNR Am J Neuroradiol 2004;25:539-44

19. Chappell MH, Ulug AM, Zhang L, et al. Distribution of microstructural damage in the brains of professional boxers: a diffusion MRI study. J Magn Reson Imaging 2006;24:537-42

20. Jezzard P, Balaban RS. Correction for geometric distortion in echo planar images from B0 field variations. Magn Reson Med 1995;34:65-73

21. Sakaie KE, Shin W, Curtin KR, et al. Method for improving the accuracy of quantitative cerebral perfusion imaging. J Magn Reson Imaging 2005;21:512-19

22. Basser PJ, Mattiello J, LeBihan D. MR diffusion tensor spectroscopy and imaging. Biophys J 1994;66:259-67

23. Basser PJ, Pierpaoli C. Microstructural and physiological features of tissues elucidated by quantitative-diffusion-tensor MRI. J Magn Reson 1996;111:209-19

24. Jenkinson M, Smith S. A global optimisation method for robust affine registration of brain images. Med Image Analysis 2001;5: $143-56$

25. Rutgers DR, Fillard P, Paradot G, et al. Diffusion tensor imaging characteristics of the corpus callosum in mild, moderate, and severe traumatic brain injury. AJNR Am J Neuroradiol 2008;29: 1730-35

AJNR Am J Neuroradiol 35:285-90 Feb 2014 www.ajnr.org 
26. Schaefer PW, Huisman TA, Sorensen AG, et al. Diffusion-weighted MR imaging in closed head injury: high correlation with initial Glasgow Coma Scale score and score on modified Rankin Scale at discharge. Radiology 2004;233:58-66

27. Wilde EA, Chu Z, Bigler ED, et al. Diffusion tensor imaging in the corpus callosum in children after moderate to severe traumatic brain injury. J Neurotrauma 2006;23:1412-26

28. Farbota KD, Bendlin BB, Alexander AL, et al. Longitudinal diffusion tensor imaging and neuropsychological correlates in traumatic brain injury patients. Front Hum Neurosci 2012;6:160

29. Wang JY, Bakhadirov K, Abdi H, et al. Longitudinal changes of structural connectivity in traumatic axonal injury. Neurology 2011;77: $818-26$

30. Kraus MF, Susmaras T, Caughlin BP, et al. White matter integrity and cognition in chronic traumatic brain injury: a diffusion tensor imaging study. Brain 2007;130:2508-19

31. Cubon VA, Putukian M, Boyer C, et al. A diffusion tensor imaging study on the white matter skeleton in individuals with sports-related concussion. J Neurotrauma 2011;28:189-201

32. Zhang K, Johnson B, Pennell D, et al. Are functional deficits in concussed individuals consistent with white matter structural alterations: combined FMRI \& DTI study. Exp Brain Res Experimentelle Hirnforschung 2010;204:57-70

33. Bazarian JJ, Zhong J, Blyth B, et al. Diffusion tensor imaging detects clinically important axonal damage after mild traumatic brain injury: a pilot study. J Neurotrauma 2007;24:1447-59

34. Wilde EA, McCauley SR, Hunter JV, et al. Diffusion tensor imaging of acute mild traumatic brain injury in adolescents. Neurology 2008;70:948-55

35. McAllister TW, Ford JC, Ji S, et al. Maximum principal strain and strain rate associated with concussion diagnosis correlates with changes in corpus callosum white matter indices. Ann Biomed Eng 2012;40:127-40

36. Mayer AR, Ling J, Mannell MV, et al. A prospective diffusion tensor imaging study in mild traumatic brain injury. Neurology 2010; 74:643-50

37. Ling JM, Pena A, Yeo RA, et al. Biomarkers of increased diffusion anisotropy in semi-acute mild traumatic brain injury: a longitudinal perspective. Brain 2012;135:1281-92

38. Wilde EA, McCauley SR, Barnes A, et al. Serial measurement of memory and diffusion tensor imaging changes within the first week following uncomplicated mild traumatic brain injury. Brain Imaging Behav 2012;6:319-28

39. Chepuri NB, Yen YF, Burdette JH, et al. Diffusion anisotropy in the corpus callosum. AJNR Am J Neuroradiol 2002;23:803-08

40. Pfefferbaum A, Sullivan EV. Increased brain white matter diffusivity in normal adult aging: relationship to anisotropy and partial voluming. Magn Reson Med 2003;49:953-61

41. Ardekani S, Kumar A, Bartzokis G, et al. Exploratory voxel-based analysis of diffusion indices and hemispheric asymmetry in normal aging. Magn Reson Imaging 2007;25:154-67

42. Giorgio A, Santelli L, Tomassini V, et al. Age-related changes in grey and white matter structure throughout adulthood. NeuroImage 2010;51:943-51

43. Mabbott DJ, Noseworthy MD, Bouffet E, et al. Diffusion tensor imaging of white matter after cranial radiation in children for medulloblastoma: correlation with IQ. Neuro-Oncology 2006;8: $244-52$

44. Yu C, Li J, Liu Y, et al. White matter tract integrity and intelligence in patients with mental retardation and healthy adults. NeuroImage 2008;40:1533-41

45. Xu J, Li Y, Lin H, et al. Body mass index correlates negatively with white matter integrity in the fornix and corpus callosum: a diffusion tensor imaging study. Hum Brain Mapp 2013;34: $1044-52$

46. McCrea M, Hammeke T, Olsen G, et al. Unreported concussion in high school football players: implications for prevention. Clin J Sport Med 2004;14:13-17 